\title{
Twin studies advance the understanding of gene-environment interplay in human nutrigenomics
}

\author{
Tess Pallister, Tim D. Spector* and Cristina Menni \\ Department of Twin Research and Genetic Epidemiology, King's College London, St Thomas' Hospital, \\ Westminster Bridge Road, London SE1 7EH, UK
}

\begin{abstract}
Investigations into the genetic architecture of diet-disease relationships are particularly relevant today with the global epidemic of obesity and chronic disease. Twin studies have demonstrated that genetic makeup plays a significant role in a multitude of dietary phenotypes such as energy and macronutrient intakes, dietary patterns, and specific food group intakes. Besides estimating heritability of dietary assessment, twins provide a naturally unique, case-control experiment. Due to their shared upbringing, matched genes and sex (in the case of monozygotic (MZ) twin pairs), and age, twins provide many advantages over classic epidemiological approaches. Future genetic epidemiological studies could benefit from the twin approach particularly where defining what is 'normal' is problematic due to the high inter-individual variability underlying metabolism. Here, we discuss the use of twins to generate heritability estimates of food intake phenotypes. We then highlight the value of discordant MZ pairs to further nutrition research through discovery and validation of biomarkers of intake and health status in collaboration with cutting-edge omics technologies.
\end{abstract}

\section{Key words: Twins: Nutrition: Heritability: Omics}

\section{Introduction}

Early nutrition studies on twins and multi-fetal pregnancies focused on the side effects of the pregnancy, achieving adequate maternal nutrition during gestation, and on the implications for the health of the growing fetuses ${ }^{(1)}$. The womb, however, is a solitary internal environment, where nutritional parameters can be measured with more ease. Once twins enter the world, where a myriad of external environmental influences on different phenotypes can be assessed, twins provide us with the unique ability to accurately determine heritability of certain complex traits, such as dietary phenotypes. In this context, dietary phenotypes broadly refer to the observable and measurable foods, and their constituents, consumed by individuals or groups. Indeed, twin studies have proven that genetic makeup plays a significant role in dietary intakes ${ }^{(2)}$. Moreover, twins have the advantage of limiting inter-individual variability as they are raised in a similar environment and have matched age, genes and sex (for monozygotic (MZ) pairs). They therefore represent a natural matched casecontrol experiment.

Omics technologies allow for a systems approach to nutrition research that encompasses the primary level of
DNA sequence (genomics), gene expression (transcriptomics) and epigenome, to the intermediate phenotypes (proteome, metabolome and microbiome), finally to clinical endpoints $^{(3)}$. Food constituents may influence each of these areas, but their effect is difficult to ascertain due to imprecise dietary assessment methods. Omics techniques will help ameliorate this issue, through food intake biomarker discovery largely within the intermediate phenotypes, primarily the metabolome ${ }^{(4)}$. Once biomarkers of intake have been validated, in conjunction with currently utilised intake and known biomarker measures, reliable associations between genomic and transcriptomic data can be made. This will allow for identification of groups susceptible to detrimental dietary patterns, gene-diet interactions and diet-disease associations, which combined will conjure an accurate depiction of a 'healthy' phenotype ${ }^{(3)}$.

In the present review, we first discuss the usage of twins for generating heritability estimates of food intake phenotypes, including energy and macronutrient intakes, dietary patterns and specific food group intakes. We then highlight the value of discordant MZ pairs, for furthering nutrition research through discovery and validation of biomarkers

Abbreviation: MZ, monozygotic.

*Corresponding author: Tim D. Spector, fax +44 207188 6761, email tim.spector@kcl.ac.uk 
of intake and health status in conjunction with cutting-edge omics technologies.

Heritability of food intake phenotypes: the usage of twins for heritability estimates of intakes

The classic twin design allows for a natural experiment that exploits the difference in genetic relatedness between $\mathrm{MZ}$ and dizygotic (DZ) twins to estimate the degree to which phenotypic variability, such as food or nutrient intake, is explained by genetic and environmental factors. Heritability is defined as the degree of total phenotypic variance due to genetic variation and is relevant only to groups or populations and not at the individual level ${ }^{(5)}$.

\section{Heritability of energy and macronutrient intakes}

Accurate assessments of the genetic influence on energy intakes are crucial as these provide a baseline assessment for which genes influence total food intakes ${ }^{(6)}$, therefore justifying genotype-specific dietary intervention strategies. Furthermore, the degree to which macronutrient intakes are genetically determined may have significant implications for the suspected role of elevated intakes of energy-dense foods (i.e. those containing high concentrations of fat and refined carbohydrates) to the obesity epidemic ${ }^{(7)}$. Therefore, multiple twin studies have evaluated these components through various dietary assessment methods (Fig. 1(a); online Supplementary Table S1).

Studies of different size and quality have estimated heritability of different energy and macronutrient intakes to lie between 8 and $70 \%{ }^{(6,8-14)}$. Beginning from a young age, infant twins have demonstrated that the genetic component provides a small, albeit significant, effect on energy and macronutrient intakes (8 to $12 \%)^{(9)}$. As children age and they become less dependent on their parents, the genetic effect on energy and macronutrient intakes appears to intensify, as suggested by findings in 11 - to 13-year-old US twins (31 to $48 \%)^{(8)}$.

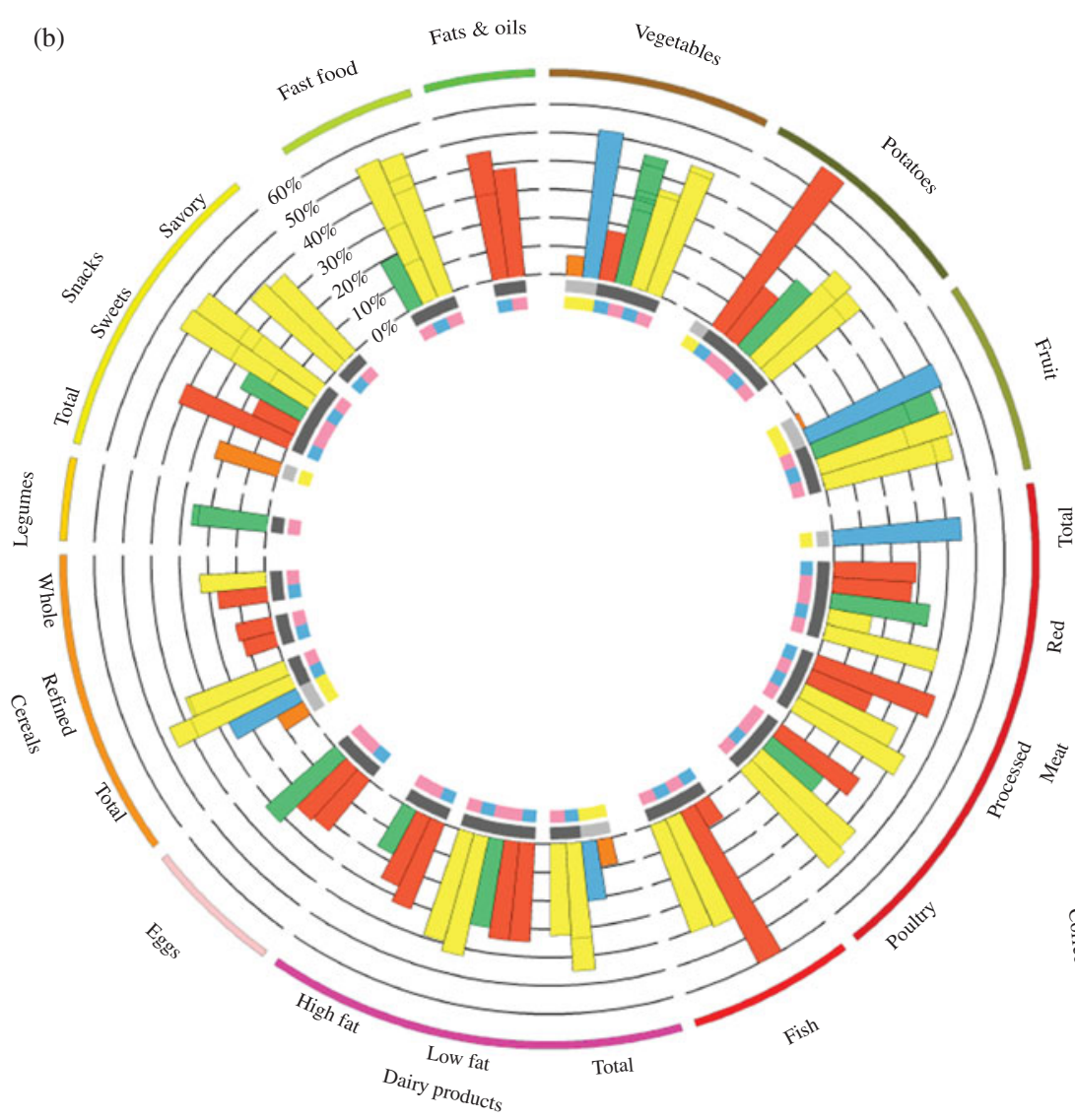

(a)
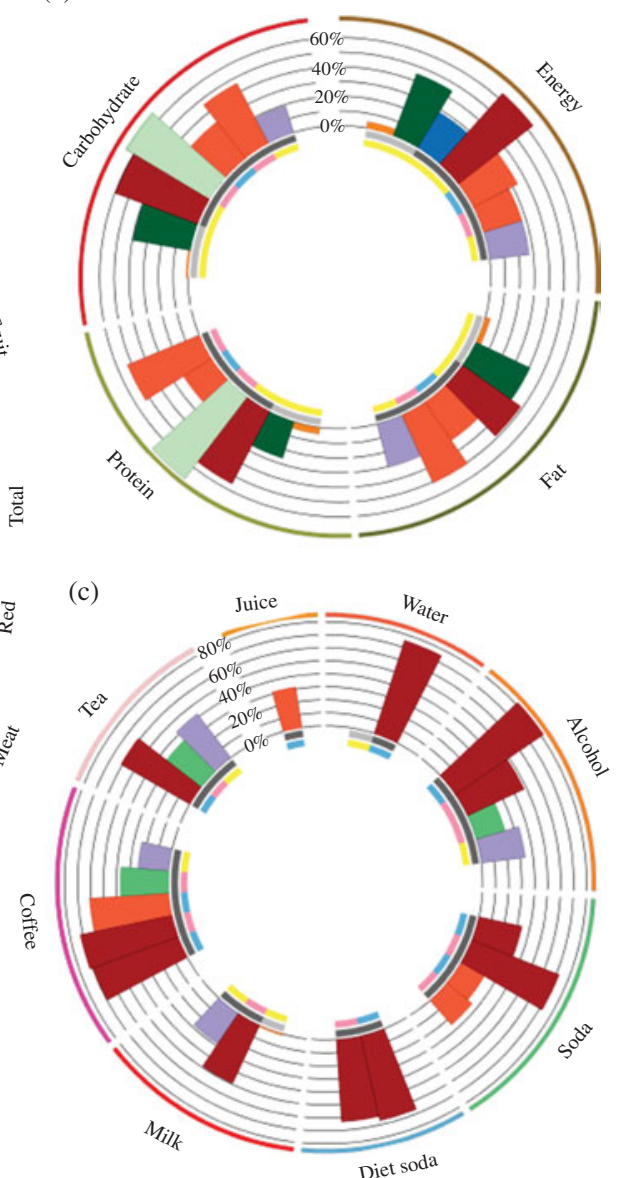

Fig. 1. Estimated heritabilities of dietary intake phenotypes: (a) energy and macronutrients; (b) foods; (c) beverages. Intake heritabilities presented are significant findings from previous twin studies (online Supplementary Tables S1, S3 and S4). Heritability histograms are colour coded according to study. From clockwise, histograms are grouped according to age; the first line below the histogram denotes this: light grey, children; dark grey, adults. Within each age group, histograms were grouped according to accuracy of the dietary assessment method used, from most accurate (for example, $2 \mathrm{~d}$ buffet-style meal intervention) to least accurate (for example, sixty-seven-item FFQ). The second line below the histograms indicates sex: blue, male; pink, female; yellow, combined. (A colour version of this figure can be found online at http://www.journals.cambridge.org/nrr). 
This pattern is similar to other health-related phenotypes, including $\mathrm{BMI}^{(15)}$, which has been recently confirmed through a more complex longitudinal genome-wide complex trait analysis for determining DNA-based heritability ${ }^{(16)}$. Heritability of dietary energy and macronutrients in adults has been found to vary widely: for energy (32 to $65 \%$ ); for fats (35 to $53 \%$ ); carbohydrates ( 25 to $67 \%$ ); and proteins (28 to $70 \%)^{(6,11,12,14)}$. Most recently, an extensive study of adult Danish twins demonstrated inheritance of energy and energy-adjusted macronutrient intakes to range from 28 to $55 \%{ }^{(12)}$. Although genetic effects on macronutrient intakes were more substantial in men (49 to $55 \%$ ) than women ( 28 to $36 \%$ ), they were not different between macronutrients. In support of this, two other twin studies suggested the same genetic mechanisms governing energy intakes $^{(7)}$ influence macronutrient intakes ${ }^{(17,18)}$.

The lack of evidence to date for independent sets of genes influencing macronutrient intakes from those genes governing energy intakes has focused attention on whether dietary energy density is genetically determined, with one study suggesting substantial genetic influence ${ }^{(19)}$, and another not ${ }^{(12)}$. Despite this, in the latter Danish study, factors related to dietary energy density, including fibre, glycaemic index and the glycaemic load, were found to be significantly heritable both in women (49, 36 and $33 \%$, respectively) and men (41, 30 and $25 \%$, respectively). The dependence of these phenotypes on the constituents of the whole diet suggests that genetic effects on these phenotypes may be reflective of variable dietary patterns.

\section{Heritability of dietary patterns}

Dietary patterns are identified by two primary methods: empirically derived or a priori ${ }^{(20)}$. Empirically derived dietary patterns employ statistical methods to identify natural groupings of intakes of food items (for example, principal component analysis or cluster analysis ${ }^{(20)}$ ). A priori dietary patterns are generated based on adherence to diet parameters previously associated with health outcomes or physiological states, such as the Mediterranean diet score. A key advantage to using dietary patterns is the translatable results that encompass the degree to which genetics influence the global diet.

Two twin studies on children using empirically derived dietary patterns generated highly inconsistent heritability estimates despite reasonable sample size (online Supplementary Table S2) ${ }^{(21,22)}$. In a study of US 7-year-olds ( $n$ 792) heritability estimates of food pattern intakes obtained by $24 \mathrm{~h}$ recalls were stronger in boys than girls, ranging from 12 to $79 \%$ and 20 to $56 \%$, respectively ${ }^{(22)}$. In another study of $4-$ to 5-year-old UK twin pairs ( $n$ 214) heritability of food preference was measured through the use of a modified ninety-five-item 'liking' FFQ administered to parents ${ }^{(21)}$. Heritability ranged from $20 \%$ (desserts) to $78 \%$ (meat and fish) on food liking-disliking groups. It is difficult to make valid comparisons between these two studies as the dietary assessment methods used measure different outcomes (i.e. intakes $v$. liking), although it has been found that exposure to foods generally encourages liking and intakes in children ${ }^{(23)}$. This suggests that environmental influences may override genetic predispositions to food pattern intakes in children, which has implications for combating food neophobia, a highly heritable and perhaps evolutionarily important trait ${ }^{(24)}$.

Contrary to studies in children, studies in adults have generated relatively stable heritability estimates for food pattern intakes across patterns and sexes. 'Healthy' dietary patterns, characterised by high intakes of vegetables, fruits and whole grains and low intakes of fatty foods of animal origin and simple carbohydrates, have heritability estimates ranging from 33 to $54 \%$, while 'unhealthy' patterns ranged from 33 to $50 \%$ (online Supplementary Table S2) ${ }^{(25-29)}$. Furthermore, a study which utilised a twins-of-mistakenzygosity approach (a useful method to control for potential equal environmental assumption bias ${ }^{(30)}$ ) in US female twins ( $n$ 700) found additive genetic effects to account for $50 \%$ of the variability in healthy pattern intakes, while an unhealthy diet pattern was not significantly heritable ${ }^{(28)}$. A healthy diet is more phenotypically similar to diets that man has consumed through millennia and evolved with; it is only very recently that high-energydense foods have been readily available, and as such it makes sense that the variation in this diet may be more genetically influenced in some groups ${ }^{(31)}$. One study conducted on an older age group ( $\geq 50$ years) determined additive genetic effects to account for $33 \%$ of the variability of consumption frequency for both healthy and unhealthy patterns ${ }^{(29)}$.

\section{Individual foods and food group heritabilities}

A number of recent studies have estimated the genetic effect of food and beverage intakes in human subjects (Figs. 1(b) and 1(c); online Supplementary Tables S3 and S4), generating wide-ranging heritability estimates across food groups, particularly for children ${ }^{(9,32)}$. Findings from the UK Gemini cohort of infants and children aged 21 months to 3 years have demonstrated that age effects on heritability of food group intakes are evident even in the early years ${ }^{(9,32)}$. Intakes for 21-month-old children were minimally heritable for food groups ranging from $9 \%$ (potatoes) to $17 \%$ (dairy products). However, preference for food groups was highly heritable in 3-year-old children, ranging from $27 \%$ (dairy products) to $54 \%$ (vegetables). It should be noted that heritability estimates in the older children were calculated from age- and sex-adjusted residuals, an approach which may have prevented inflation of shared environmental effects ${ }^{(33)}$. The study on infants did not use this approach, perhaps resulting in an underestimation of the genetic effects on food group intakes.

Recent studies in adults have also confirmed that there is a genetic component to many food type intakes. Danish twins demonstrated a large degree of variation in 
calculated heritabilities overall ranging from $17 \%$ (fish) to $68 \%$ (potatoes) in men, and $20 \%$ (whole-grain cereal) to $61 \%$ (fish) in women ${ }^{(12)}$. Similar results were seen in UK female twins where heritabilities ranged from $8 \%$ (refined grains) to $46 \%$ (garlic) $^{(27)}$. Furthermore, the grouping of fruit and vegetable sources generated the highest heritability estimate in this cohort (49 \%). These authors proposed that taste perception may be a key driver of the predicted genetic influence, as foods characterised by strong tastes are the cornerstone of these groups. This observation extends to consistently substantial heritabilities for coffee as well as alcohol intakes, ranging from 29 to $73 \%$ and 28 to $82 \%$, respectively ${ }^{(12,14,27,34)}$. However, the pharmacological properties of both must be considered as alcohol dependence, as well as caffeine consumptionrelated traits, are both highly heritable (30 to $70 \%$, and 36 to $58 \%$, respectively) ${ }^{(35,36)}$ and may drive intakes up in a way independent of taste preference or a biological requirement for these items.

Taste is the strongest determinant of food choices ${ }^{(37)}$, and a recent twin study demonstrated multiple chemosensory facets of numerous food compounds to be highly heritable and associated with particular gene variants ${ }^{(38)}$. Although an investigation of this area is beyond the scope of the present review, future heritability studies of food intakes may shift the focus to food groupings characterised by specific tastes.

\section{Nutritional phenotype heritability conclusion}

In summary, twin studies have demonstrated food and macronutrient intakes to be genetically determined. However, heritability estimates are often inconsistent between studies for multiple reasons. First, heritability is only specific to the population studied, which can vary greatly, due to the vast country-specific food environments. Indeed, if a food is not readily available in a population there is no capacity for intakes to be genetically determined. Second, although beyond the scope of the present review, important evidence is available for familial and community influence on food intake (such as parenting styles, food exposures, socio-economic status), which have an important place for battling current issues, such as childhood obesity ${ }^{(39)}$ and food neophobia ${ }^{(40)}$, and diabetes ${ }^{(41)}$. Finally, methods for determining heritability are not standardised, for instance the inclusion/ exclusion of variables from the model (for example, age, country), the segregation/aggregation of sexes, and the different methods for obtaining and analysing dietary data tend to be unique for each study. The same genetic mechanisms governing energy intakes are suspected to influence macronutrient intakes. However, recent studies have assessed the impact of gene variants encoding proteins related to the complex energy intake regulatory systems under central nervous system control $^{(7)}$ on preference for macronutrient types. These studies are few on twins $^{(42,43)}$. Heritability estimates are more limited for macronutrient subtype intakes (for example, the fatty acid profile $\left.{ }^{(10,14)}\right)$, different age groups or changing physiological states (for example, pregnancy), and socially driven diets (for example, veganism). Macronutrient profiles do not account entirely for food pattern preferences that may be more determined by genetic makeup. Variation in diet patterns of adults is to a relatively high degree attributable to genetics ${ }^{(25-29)}$. Furthermore, specific food-type intakes that characterise a healthy diet pattern tend to be highly heritable in adults and in turn consist of foods that elicit distinctive taste responses (for example, garlic, fruit and vegetables) ${ }^{(27)}$.

The substantial heritability of multiple dietary phenotypes supports the concept of personalised dietary recommendations, the generation of which has so far primarily relied on the exploration of an individual's genetic makeup. However, on a molecular level, the search for genotypes predisposing individuals to particular dietary intakes is very much in its infancy. Nonetheless, the evidence generated by twin heritability studies can lead scientists to dietary phenotypes that are consistently highly heritable and demand further exploration through genome-wide association and candidate gene studies, following which twins can aid the determination of the heritable contribution of specific SNP to the dietary phenotype (as used previously for human height ${ }^{(44)}$ ).

A key issue that has long plagued nutrigenetic research arises from the inaccuracies of self-reported diet intake and food preference data and, as a result, the hunt for dietary biomarkers, objective measures of dietary intakes and nutritional status, has been hastened. These may allow for more accurate heritability calculations for food and nutrient intakes as has recently been demonstrated for salt intakes using urinary $\mathrm{Na}$ excretion, an established, valid biomarker of salt intake ${ }^{(45)}$, and could potentially be used in genetic association studies. However, the usage of biomarkers is not confined to genetic studies. It is becoming increasingly clear that for the establishment and refinement of personalised nutrition, in combination with genetic information, a whole organism, multi-systems approach must be undertaken, where the usage of biomarkers of food intakes and nutritional status will be essential. Nutrition research is now embarking on a nutriomics era, where emerging high-throughput omics technologies will substantially aid biomarker discovery and 'healthy phenotype' definition. As will be explored next, twin studies are progressively taking steps to make a major contribution in this way.

\section{The importance of twins for emerging nutriomic research}

This next section will explore how diet makes an impact on genes themselves (epigenetics), gene expression (transcriptomics), protein expression (proteomics) and metabolism (metabolomics). Integrating these technologies 
to determine a 'healthy' phenotype is the long-term goal of personalised nutrition ${ }^{(46)}$. A reoccurring issue with this type of analysis is the high degree of inter-individual variation, making associations often inconsistent ${ }^{(3)}$. As such, the usage of identical twins discordant for dietary factors or objective measures of nutrition status, matched entirely for age, sex, genetics and partially for early environmental influences, will provide an enhanced method of assessing diet and biological relationships.

Obesity, the greatest and fastest-growing health concern in the world at this time, is a highly genetic disorder, influenced by myriad complex factors ${ }^{(47)}$. However, rare obese discordant identical twin pairs provide a unique opportunity to disentangle lifestyle and environmental factors on the human system derangements induced by energy imbalance independently of genetics.

\section{Nutrimetabolomics}

Nutritional metabolomics involves the extensive chemical profiling of various tissues completed in a global manner through targeted and untargeted methods used as a complement to diet- and health-related complex systems approaches. The metabolome gives unique information into the metabolic status of an individual by providing a snapshot of the metabolic processes undertaken in a bodily system, specific organ, tissue or cell, which cannot be identified through measuring gene expression or the proteome. Nutrition and the metabolome are intimately linked in that nutrients and non-nutrient food constituents supply metabolites; however, these contributions make the dietary influence on the metabolome all the more difficult to ascertain ${ }^{(48)}$. Despite this, dietary pattern and food intakes generated through self-reported intake data have identified novel biomarkers within the metabolome ${ }^{(49-51)}$, validating this approach for use in epidemiological studies. However, the genetic influence on metabolite levels is wide-ranging ${ }^{(52)}$, therefore novel usage of the twin model provides an ideal method for determining the nutritional impact on metabolites, through segregating the nongenetic component. Recently, a nutri-metabolomic study conducted on twins further confirmed self-reported intake associations with metabolites ${ }^{(53)}$. Female UK twins completed a 131-item FFQ that generated dietary constituents and intake patterns that were found to associate with forty-two metabolite levels in the larger twin population. MZ twins discordant for dietary intakes were then identified and utilised to replicate results, confirming the utility of this $\operatorname{method}^{(4)}$.

Lipidomics, a branch of metabolomics, was used to characterise environmental and lifestyle-induced changes to the global serum lipid profile in fourteen healthy, young MZ obesity-discordant twin pairs ${ }^{(54)}$. Levels of lysophosphatidylcholines, lipids associated with inflammation $^{(55)}$ and atherogenesis ${ }^{(56)}$, were found to be elevated in obese co-twins, with concommitant decreases in antioxidant ${ }^{(57)}$ ether phospholipids. Moreover, despite the young ages of the obese co-twins (24 to 27 years), these lipid profile changes were associated with insulin resistance.

The metabolome is highly complex and inter-individual variation is high for a multitude of reasons ${ }^{(58)}$, thus factoring solely nutritional intake into nutrimetabolomic studies is problematic and the relative importance of blood and urine levels is unclear. For instance, the gut microbiota has been determined to account for $10 \%$ of the variability in the plasma metabolome in rodents ${ }^{(59)}$. Although estimates for humans are unknown at this time, regional variation in human metabolomic profiles has been attributed in part to gut microbes ${ }^{(60)}$. In turn, the gut microbiota carry out processes in the digestion and handling of nutrients, some of which are essential for optimal nutrition, before entering the host metabolome ${ }^{(60)}$. Emerging methods for direct measurement of the intestinal metabolome will help to unravel effects of the microbiome on the host metabolome ${ }^{(61)}$.

\section{Microbiome}

The human gut microbiome is estimated to contain hundreds of bacterial species, with upwards of 536000 bacterial genes among them ${ }^{(62)}$. For years, dietary constituents, such as pre- and probiotics, have been consumed for their proposed health benefits postulated to be through modulation of the gut microbial population ${ }^{(63)}$. More recently, vegetarian and omnivorian dietary patterns have been associated with differential gut microbial species in a Slovenian population study ${ }^{(64)}$. In a recent study, twins discordant for obesity have demonstrated significantly different gut microbial species composition ${ }^{(65)}$. Lower BMI was associated with increased primary fibre degraders, while higher BMI subjects displayed an abundant network of butyrate producers; as such it was suggested that a shift in fermentation patterns near the end of the colon may be influencing energy homeostasis, although this study neglected to investigate the dietary impact on the microbial species population.

A recent analysis on Finnish MZ twin pairs concordant ( $n$ 9) and discordant ( $n$ 11) for BMI found dietary energy intakes and constituents to be more influential on microbial populations than BMI group ${ }^{(66)}$. Specifically, stool bacterial counts were significantly influenced by intakes of energy, MUFA and $n-3$ and $n-6$ PUFA, and soluble fibre intakes. Furthermore, profiles of Bacteroides spp. were very similar in co-twins consuming the same amount of energy or saturated fats than twins discordant for intakes. These findings suggest that the obesity status per se is less influential on microbial species population and, rather, it is the content of the diet (i.e. high energy and fat) mediating important changes, highlighting the essential need to incorporate dietary variables into obesity research. The role of host genetics on the microbiome 
composition is still unclear and larger studies (which are ongoing) are needed to provide clarity ${ }^{(67)}$.

Recent findings suggest that the microbial species signature has the potential to promote and aggravate an obesity phenotype and may interact synergistically through diet $\times$ microbiota interactions. In a unique study, transplantation of faecal samples from US female lean and obese co-twins (three dizygotic; one MZ) into germ-free mice revealed that the obese ( $\mathrm{Ob}$ mice) compared with lean (Ln mice) co-twin's microbiota significantly increased adiposity and metabolic derangements in mice ${ }^{(68)}$. When co-housed with Ln mice, Ob mice adiposity and metabolic effects were reduced, suspected to be a result of Bacteroidetes translocation from Ln to $\mathrm{Ob}$ mice. When fed a diet based on the lower tertile of saturated fats and upper tertile of fruits and vegetables of the US National Health and Nutrition Examination Survey (NHANES), Ob mice adiposity was sustained, but relieved when co-housed with Ln mice. Moreover, when compared with co-housing with normal chow, the healthier diet aided the success of Ln to Ob mice bacterial invasion. When supplied with an unhealthy diet containing the upper tertile of saturated fats with the lower tertile of fruits and vegetables from the NHANES survey, both $\mathrm{Ln}$ and $\mathrm{Ob}$ mice presented with significantly increased adiposity, mitigating transmissible effects. These results show the potentially causal role of the microbiome composition and potential for its manipulation.

\section{Epigenetics}

Epigenetic variation is another source of phenotypic variation and discordance in twins ${ }^{(69)}$. Epigenetics involves the changes to marks on the genome that are copied from one cell generation to the next, which may alter gene expression, but does not involve modifications of the DNA sequence. Three specific, albeit inter-related, epigenetic mechanisms that control the magnitude and timing of gene expression during cellular differentiation and fetal development, as well as throughout life, are possibly modified by dietary factors (for a summary, see Canani et $a l{ }^{(70)}$ ). These include DNA methylation, histone modifications and noncoding microRNA ${ }^{(71,72)}$. However, the bulk of the evidence for these mechanisms originates from animal studies.

Identical twins display large epigenetic differences at birth $^{(73)}$. On diet, numerous studies have emerged focusing on the developmental origins of adult disease (Barker) hypothesis $^{(74)}$. This involves the influence of detrimental maternal nutrition, and other lifestyle factors, contributing to intra-uterine growth retardation, and subsequent premature birth and/or low birth weight. This flawed in utero environment may activate fetal epigenetic events, inducing long-term changes in expression of genes encoding proteins responsible for metabolic processes, potentially leading to chronic diseases in adulthood. Data are still accumulating on the extent of this effect, and no good longitudinal studies yet exist. An early study found young
MZ twins to be more epigenetically alike than adult twin pairs, and this distinction extended to gene expression profiles ${ }^{(75)}$. In addition, twin birth weight has been found to be less influential on adult body adiposity than an obesogenic postnatal environment ${ }^{(76)}$. This suggests that environmental and genetic events throughout life, not only during the early years, influence the epigenome and gene expression.

Recently, an MZ twin candidate gene study demonstrated that SLC6A4 promoter, a serotonin transporter gene, hypermethylation in blood leucocytes was significantly associated with increased adiposity measures including BMI, body weight and waist circumference ${ }^{(77)}$ Interestingly, although causality could not be determined, the serotonergic system is involved in the complex energy-regulatory processes governing energy intakes. A lone study on epigenetic differences in $\mathrm{MZ}$ twin pairs discordant for BMI ( $n$ 16) found no significant differences in gene methylation at differentially methylated regions most associated with abnormal growth syndromes, many characterised by severe obesity ${ }^{(78)}$. However, only DNA extracted from saliva samples was used for this study and recent findings suggest that DNA methylation is tissue specific $^{(79)}$; therefore analysis of different tissues may provide contrasting findings. New high-throughput epigenome sequencing in connection with BMI has recently provided promising findings ${ }^{(80)}$, in conjuction with transcriptomics; this may provide more definitive answers as coverage so far has been poor ${ }^{(80)}$.

\section{Transcriptomics}

The purposes of transcriptomics for nutrition research include the provision of biologically relevant mechanisms for effects of particular nutrients or diet patterns, confirming alterations in genes, proteins or metabolites in pre-disease state which may serve as biomarkers, and, finally, identifying and elucidating nutrient-regulated pathways ${ }^{(81)}$. With these purposes in mind, the use of twins with their shared genetic background can add significant strength to transcriptomic studies. Despite this, twin studies have to date largely focused on defining gene expression phenotypes related to metabolic derangements, particularly those related to obesity.

An analysis of global gene expression patterns in the adipose tissue of obesity-discordant MZ twin pairs has demonstrated marked changes induced by acquired obesity $^{(82)}$, such as a reduction in mitochondrial DNA copy number, which may impair oxidative phosphorylation. Additionally, a significant down-regulation of mitochondrial branched-chain amino acid catabolism, a process commonly activated during adipocyte differentiation in conjunction with PPAR $\gamma$ and metabolites of oxidative energy processes, has been observed ${ }^{(83)}$. These derangements of energy metabolism in adipose tissue were associated with liver fat accumulation, insulin 
resistance and hyperinsulinaemia in obese co-twins, providing significant evidence for the influence of lifestyle to obesity-related metabolic derangements. A limited analysis of dietary intake demonstrated that lower amounts of MUFA and PUFA were consumed by obese co-twins. This could be of interest, as unsaturated fatty acids are potent activators of $P P A R \alpha$ and $P P A R \gamma$ by acting as nuclear transcription factors ${ }^{(84)}$. Pathways involved in inflammatory and immune responses were highly overexpressed in adipose tissue in the former twin study, particularly the pro-inflammatory cytokine osteopontin ${ }^{(85)}$.

\section{Proteomics}

Proteomics remains the most complex challenge to omic studies, due to the expanse of the human protein expression profile and to the uncertainty of how many proteins exist. For perspective, the human genome includes greater than 25000 genes $^{(86)}$, while the human proteome includes at least 100000 proteins, compounded by various forms and variants $^{(87)}$. To date, few proteomic studies have been conducted on twins. One study quantified serum protein levels by antibody arrays and determined variation attributable to familial factors to be minimal (12\%), while experimental variation was quite large (63\%), accentuating the importance of considering the latter when designing and evaluating studies for biomarker discoveries utilising high-throughput methods ${ }^{(88)}$. To date, no twin or obese co-twin studies have incorporated dietary factors using these methods. Whereas global protein expression levels are available, protein structure and function studies are far from being understood, yet have huge future potential ${ }^{(3)}$.

\section{Conclusion}

The interconnectedness between each omic presented in this review is evident. A systems biological approach that incorporates multiple omics methods for phenotype definition for use in nutriomic studies is much needed $^{(89)}$. The principle central to this approach is that the whole organism will provide a more accurate view than the sum of its parts, or, rather, the entire system has definitive characteristics which will not be replicated by simply adding the effects ${ }^{(90)}$. However, a primary setback for amalgamating these methods is the inability to define what is 'normal' due to the significant inter-individual variability underlying metabolic processes ${ }^{(3)}$. Furthermore, it has been suggested that many omics vary relatively little over time within individuals but significantly between individuals compounded by factors such as age, BMI and sex $^{(91)}$. To aid in discerning these effects, longitudinal phenotypic information from twin registries will be a critical resource for molecular dietary studies of the future ${ }^{(92)}$. The studies presented in this review fall short of this view of an integrated technology-driven approach and focus rather on omics in isolation, as this is where the current research stands. To move forward twin cohorts need to make a collaborative effort, collecting extensive dietary data in conjunction with multiple omics, undertaking complex statistical analyses, while contributing findings to international proposed databases such as the Nutritional Phenotype database ${ }^{(93)}$.

Identifying individuals susceptible to poor dietary habits and defining an (un)healthy phenotype are the overarching aims of nutrigenetic and nutrigenomic research. Twins have provided valuable evidence that many dietary intakes are influenced by genetics, validating further nutrigenetic research and future dietary counselling which targets this domain. Early studies of twins for nutriomic studies have primarily been for unhealthy phenotype definition and used a single omic, while being less inclusive of diet. However, future studies need to begin to incorporate dietary factors to fill a void in this research area. However, accurate dietary assessment methods are problematic ${ }^{(94)}$. As twins have demonstrated, metabolites are significantly correlated with dietary constituents, and could in the future be used as surrogates, as well as other techniques (for example, the microbiome) ${ }^{(95,96)}$. Twin studies will remain an important and integral part of nutritional research now and in the future.

\section{Acknowledgements}

Twins UK was funded by the Wellcome Trust; European Community's Seventh Framework Programme (FP7/ 2007-2013). The study also receives support from the National Institute for Health Research (NIHR) BioResource Clinical Research Facility and Biomedical Research Centre based at Guy's and St Thomas' NHS Foundation Trust and King's College London. T. D. S. is holder of an ERC Advanced Principal Investigator award.

All authors contributed, read and approved the final version.

None of the authors had any conflicts of interest.

\section{Supplementary material}

To view supplementary material for this article, please visit http://dx.doi.org/10.1017/S095442241400016X

\section{References}

1. Brown JE \& Carlson M (2000) Nutrition and multifetal pregnancy. J Am Diet Assoc 100, 343-348.

2. Rankinen T \& Bouchard C (2006) Genetics of food intake and eating behavior phenotypes in humans. Annu Rev Nutr 26, 413-434.

3. Kussmann M, Raymond F \& Affolter M (2006) OMICS-driven biomarker discovery in nutrition and health. J Biotechnol 124, 758-787.

4. Zivkovic AM \& German JB (2009) Metabolomics for assessment of nutritional status. Current Opin Clin Nutr Metab Care 12, 501-507. 
5. Visscher PM, Hill WG \& Wray NR (2008) Heritability in the genomics era - concepts and misconceptions. Nat Rev Genet 9, 255-266.

6. de Castro JM (1993) Genetic influences on daily intake and meal patterns of humans. Physiol Behav 53, 777-782.

7. Guyenet SJ \& Schwartz MW (2012) Clinical review: regulation of food intake, energy balance, and body fat mass: implications for the pathogenesis and treatment of obesity. J Clin Endocrinol Metab 97, 745-755.

8. Liu J, Tuvblad C, Raine A, et al. (2013) Genetic and environmental influences on nutrient intake. Genes Nutr $\mathbf{8}$, 241-252.

9. Pimpin L, Ambrosini GL, Llewellyn CH, et al. (2013) Dietary intake of young twins: nature or nurture? Am J Clin Nutr $\mathbf{9 8}$, $1326-1334$

10. Heller RF, O'Connell DL, Roberts DC, et al. (1988) Lifestyle factors in monozygotic and dizygotic twins. Genet Epidemiol 5, 311-321.

11. Wade J, Milner J \& Krondl M (1981) Evidence for a physiological regulation of food selection and nutrient intake in twins. Am J Clin Nutr 34, 143-147.

12. Hasselbalch AL, Heitmann BL, Kyvik KO, et al. (2008) Studies of twins indicate that genetics influence dietary intake. J Nutr 138, 2406-2412.

13. Aden DP, Fogel A, Plotkin S, et al. (1979) Controlled synthesis of HBsAg in a differentiated human liver carcinomaderived cell line. Nature 282, 615-616.

14. Hur YM, Bouchard TJ Jr \& Eckert E (1998) Genetic and environmental influences on self-reported diet: a rearedapart twin study. Physiol Behav 64, 629-636.

15. Dubois L, Ohm Kyvik K, Girard M, et al. (2012) Genetic and environmental contributions to weight, height, and BMI from birth to 19 years of age: an international study of over 12,000 twin pairs. PLOS ONE 7, e30153.

16. Llewellyn CH, Trzaskowski M, Plomin R, et al. (2014) From modeling to measurement: developmental trends in genetic influence on adiposity in childhood. Obesity (Silver Spring) 22, 1756-1761.

17. de Castro JM (1993) Independence of genetic influences on body size, daily intake, and meal patterns of humans. Physiol Behav 54, 633-639.

18. Faith MS, Rha SS, Neale MC, et al. (1999) Evidence for genetic influences on human energy intake: results from a twin study using measured observations. Behav Genet 29, $145-154$.

19. de Castro JM (2006) Heredity influences the dietary energy density of free-living humans. Physiol Behav 87, 192-198.

20. Hu FB (2002) Dietary pattern analysis: a new direction in nutritional epidemiology. Curr Opin Lipidol 13, 3-9.

21. Breen FM, Plomin R \& Wardle J (2006) Heritability of food preferences in young children. Physiol Behav 88, 443-447.

22. Faith MS, Rhea SA, Corley RP, et al. (2008) Genetic and shared environmental influences on children's 24-h food and beverage intake: sex differences at age 7 y. Am J Clin Nutr 87, 903-911.

23. Wardle J, Sanderson S, Gibson EL, et al. (2001) Factoranalytic structure of food preferences in four-year-old children in the UK. Appetite 37, 217-223.

24. Cooke LJ, Haworth CM \& Wardle J (2007) Genetic and environmental influences on children's food neophobia. Am J Clin Nutr 86, 428-433.

25. van den Berg L, Henneman P, Willems van Dijk K, et al. (2013) Heritability of dietary food intake patterns. Acta Diabetol 50, 721-726.

26. Keskitalo K, Silventoinen K, Tuorila H, et al. (2008) Genetic and environmental contributions to food use patterns of young adult twins. Physiol Behav 93, 235-242.
27. Teucher B, Skinner J, Skidmore PM, et al. (2007) Dietary patterns and heritability of food choice in a UK female twin cohort. Twin Res Hum Genet 10, 734-748.

28. Gunderson EP, Tsai AL, Selby JV, et al. (2006) Twins of mistaken zygosity (TOMZ): evidence for genetic contributions to dietary patterns and physiologic traits. Twin Res Hum Genet 9, 540-549.

29. van den Bree MB, Eaves LJ \& Dwyer JT (1999) Genetic and environmental influences on eating patterns of twins aged $>/=50$ y. Am J Clin Nutr 70, 456-465.

30. Scarr S (1968) Environmental bias in twin studies. Eugen $Q$ 15, 34-40.

31. Breslin PA (2013) An evolutionary perspective on food and human taste. Curr Biol 23, R409-R418.

32. Fildes A, van Jaarsveld CH, Llewellyn CH, et al. (2014) Nature and nurture in children's food preferences. Am J Clin Nutr 99, 911-917.

33. McGue M \& Bouchard TJ Jr (1984) Adjustment of twin data for the effects of age and sex. Behav Genet 14, 325-343.

34. de Castro JM (1993) A twin study of genetic and environmental influences on the intake of fluids and beverages. Physiol Behav 54, 677-687.

35. Agrawal A \& Lynskey MT (2008) Are there genetic influences on addiction: evidence from family, adoption and twin studies. Addiction 103, 1069-1081.

36. Yang A, Palmer AA \& de Wit H (2010) Genetics of caffeine consumption and responses to caffeine. Psychopharmacology 211, 245-257.

37. Feeney E, O'Brien S, Scannell A, et al. (2011) Genetic variation in taste perception: does it have a role in healthy eating? Proc Nutr Soc 70, 135-143.

38. Knaapila A, Hwang LD, Lysenko A, et al. (2012) Genetic analysis of chemosensory traits in human twins. Chem Senses 37, 869-881.

39. Berge JM (2009) A review of familial correlates of child and adolescent obesity: what has the 21st century taught us so far? Int I Adolesc Med Health 21, 457-483.

40. Anzman-Frasca S, Savage JS, Marini ME, et al. (2012) Repeated exposure and associative conditioning promote preschool children's liking of vegetables. Appetite 58, 543-553.

41. Pollard SL, Zachary DA, Wingert K, et al. (2014) Family and community influences on diabetes-related dietary change in a low-income urban neighborhood. Diabetes Educ $\mathbf{4 0}$, 462-469.

42. Hasselbalch AL, Angquist L, Christiansen L, et al. (2010) A variant in the fat mass and obesity-associated gene (FTO) and variants near the melanocortin- 4 receptor gene (MC4R) do not influence dietary intake. $J$ Nutr 140, 831-834.

43. Bouchard-Mercier A, Paradis AM, Perusse L, et al. (2012) Associations between polymorphisms in genes involved in fatty acid metabolism and dietary fat intakes. $J$ Nutrigenet Nutrigenomics 5, 1-12.

44. Yang J, Benyamin B, McEvoy BP, et al. (2010) Common SNPs explain a large proportion of the heritability for human height. Nat Genet 42, 565-569.

45. Kho M, Lee JE, Song YM, et al. (2013) Genetic and environmental influences on sodium intake determined by using half-day urine samples: the Healthy Twin Study. Am J Clin Nutr 98, 1410-1416.

46. van Ommen B \& Stierum R (2002) Nutrigenomics: exploiting systems biology in the nutrition and health arena. Curr Opin Biotechnol 13, 517-521.

47. Després JP, Moorjani S, Lupien PJ, et al. (1992) Genetic aspects of susceptibility to obesity and related dyslipidemias. Mol Cellular Biochem 113, 151-169. 
48. Gibney MJ, Walsh M, Brennan L, et al. (2005) Metabolomics in human nutrition: opportunities and challenges. Am J Clin Nutr 82, 497-503.

49. O'Sullivan A, Gibney MJ \& Brennan L (2011) Dietary intake patterns are reflected in metabolomic profiles: potential role in dietary assessment studies. Am J Clin Nutr 93, 314-321.

50. Altmaier E, Kastenmuller G, Romisch-Margl W, et al. (2011) Questionnaire-based self-reported nutrition habits associate with serum metabolism as revealed by quantitative targeted metabolomics. Eur J Epidemiol 26, 145-156.

51. Guertin KA, Moore SC, Sampson JN, et al. (2014) Metabolomics in nutritional epidemiology: identifying metabolites associated with diet and quantifying their potential to uncover diet-disease relations in populations. Am J Clin Nutr 100, 208-217.

52. Suhre K, Shin SY, Petersen AK, et al. (2011) Human metabolic individuality in biomedical and pharmaceutical research. Nature $\mathbf{4 7 7}, 54-60$.

53. Menni C, Zhai G, Macgregor A, et al. (2013) Targeted metabolomics profiles are strongly correlated with nutritional patterns in women. Metabolomics 9, 506-514.

54. Pietilainen KH, Sysi-Aho M, Rissanen A, et al. (2007) Acquired obesity is associated with changes in the serum lipidomic profile independent of genetic effects - a monozygotic twin study. PloS ONE 2, e218.

55. Yang LV, Radu CG, Wang L, et al. (2005) GI-independent macrophage chemotaxis to lysophosphatidylcholine via the immunoregulatory GPCR G2A. Blood 105, 1127-1134.

56. Glass CK \& Witztum JL (2001) Atherosclerosis. the road ahead. Cell 104, 503-516.

57. Wallner S \& Schmitz G (2011) Plasmalogens the neglected regulatory and scavenging lipid species. Chem Phys Lipids 164, 573-589

58. Zulyniak MA \& Mutch DM (2011) Harnessing metabolomics for nutrition research. Curr Pharm Biotechnol 12 1005-1015.

59. Wikoff WR, Anfora AT, Liu J, et al. (2009) Metabolomics analysis reveals large effects of gut microflora on mammalian blood metabolites. Proc Nat Acad Sci U S A 106, 3698-3703.

60. Nicholson JK, Holmes E, Kinross J, et al. (2012) Host-gut microbiota metabolic interactions. Science 336, 1262-1267.

61. Ursell LK, Haiser HJ, Van Treuren W, et al. (2014) The intestinal metabolome: an intersection between microbiota and host. Gastroenterology 146, 1470-1476.

62. Qin J, Li R, Raes J, et al. (2010) A human gut microbial gene catalogue established by metagenomic sequencing. Nature 464, 59-65.

63. Collins MD \& Gibson GR (1999) Probiotics, prebiotics, and synbiotics: approaches for modulating the microbial ecology of the gut. Am J Clin Nutr 69, 1052s-1057s.

64. Matijašić BB, Obermajer T, Lipoglavšek L, et al. (2014) Association of dietary type with fecal microbiota in vegetarians and omnivores in Slovenia. Eur J Nutr $\mathbf{5 3}$ 1051-1064.

65. Tims S, Derom C, Jonkers DM, et al. (2013) Microbiota conservation and BMI signatures in adult monozygotic twins. ISME J 7, 707-717.

66. Simoes CD, Maukonen J, Kaprio J, et al. (2013) Habitual dietary intake is associated with stool microbiota composition in monozygotic twins. J Nutr 143, 417-423.

67. Turnbaugh PJ \& Gordon JI (2009) The core gut microbiome, energy balance and obesity. J Physiol 587, 4153-4158.

68. Ridaura VK, Faith JJ, Rey FE, et al. (2013) Gut microbiota from twins discordant for obesity modulate metabolism in mice. Science 341, 1241214.

69. Bell JT \& Spector TD (2012) DNA methylation studies using twins: what are they telling us? Genome Biol 13, 172.
70. Canani RB, Costanzo MD, Leone L, et al. (2011) Epigenetic mechanisms elicited by nutrition in early life. Nutr Res Rev 24, 198-205.

71. McKay JA \& Mathers JC (2011) Diet induced epigenetic changes and their implications for health. Acta Physiol 202, 103-118.

72. Zeisel SH (2009) Epigenetic mechanisms for nutrition determinants of later health outcomes. Am J Clin Nutr 89, 1488 s-1493s

73. Loke YJ, Novakovic B, Ollikainen M, et al. (2013) The Peri/ postnatal Epigenetic Twins Study (PETS). Twin Res Hum Genet 16, 13-20.

74. de Boo HA \& Harding JE (2006) The developmental origins of adult disease (Barker) hypothesis. Aust $N Z J$ Obstet Gynaecol 46, 4-14.

75. Fraga MF, Ballestar E, Paz MF, et al. (2005) Epigenetic differences arise during the lifetime of monozygotic twins. Proc Nat Acad Sci U S A 102, 10604-10609.

76. Skidmore PM, Cassidy A, Swaminathan R, et al. (2009) An obesogenic postnatal environment is more important than the fetal environment for the development of adult adiposity: a study of female twins. Am J Clin Nutr 90, 401-406.

77. Zhao J, Goldberg J \& Vaccarino V (2013) Promoter methylation of serotonin transporter gene is associated with obesity measures: a monozygotic twin study. Int $J$ Obes 37, 140-145.

78. Souren NY, Tierling S, Fryns JP, et al. (2011) DNA methylation variability at growth-related imprints does not contribute to overweight in monozygotic twins discordant for BMI. Obesity (Silver Spring) 19, 1519-1522.

79. Lokk K, Modhukur V, Rajashekar B, et al. (2014) DNA methylome profiling of human tissues identifies global and tissue-specific methylation patterns. Genome Biol 15, r54

80. Dick KJ, Nelson CP, Tsaprouni L, et al. (2014) DNA methylation and body-mass index: a genome-wide analysis. Lancet 383, 1990-1998.

81. Müller M \& Kersten S (2003) Nutrigenomics: goals and strategies. Nat Rev Genet 4, 315-322.

82. Pietilainen KH, Naukkarinen J, Rissanen A, et al. (2008) Global transcript profiles of fat in monozygotic twins discordant for BMI: pathways behind acquired obesity. PLoS Med 5, e51.

83. Hackl H, Burkard TR, Sturn A, et al. (2005) Molecular processes during fat cell development revealed by gene expression profiling and functional annotation. Genome Biol 6, R108.

84. Keller H, Dreyer C, Medin J, et al. (1993) Fatty acids and retinoids control lipid metabolism through activation of peroxisome proliferator-activated receptor-retinoid X receptor heterodimers. Proc Nat Acad Sci U S A 90, 2160-2164.

85. Daniele G, Guardado Mendoza R, Winnier D, et al. (2014) The inflammatory status score including IL- 6 , TNF- $\alpha$, osteopontin, fractalkine, MCP-1 and adiponectin underlies whole-body insulin resistance and hyperglycemia in type 2 diabetes mellitus. Acta Diabetol 51, 123-131.

86. Lander ES, Linton LM, Birren B, et al. (2001) Initial sequencing and analysis of the human genome. Nature $\mathbf{4 0 9}$, $860-921$.

87. Kussmann M, Affolter M \& Fay LB (2005) Proteomics in nutrition and health. Comb Chem High Throughput Screen 8, 679-696.

88. Kato BS, Nicholson G, Neiman M, et al. (2011) Variance decomposition of protein profiles from antibody arrays using a longitudinal twin model. Proteome Sci 9, 73

89. Norheim F, Gjelstad IM, Hjorth M, et al. (2012) Molecular nutrition research: the modern way of performing nutritional science. Nutrients 4, 1898-1944. 
90. MacLellan WR, Wang Y \& Lusis AJ (2012) Systems-based approaches to cardiovascular disease. Nat Rev Cardiol 9, 172-184.

91. Eady JJ, Wortley GM, Wormstone YM, et al. (2005) Variation in gene expression profiles of peripheral blood mononuclear cells from healthy volunteers. Physiol Genomics 22, 402-411.

92. van Dongen J, Slagboom PE, Draisma HH, et al. (2012) The continuing value of twin studies in the omics era. Nat Rev Genet 13, 640-653.

93. van Ommen B, Bouwman J, Dragsted LO, et al. (2010) Challenges of molecular nutrition research 6: the nutritional phenotype database to store, share and evaluate nutritional systems biology studies. Genes Nutr 5, 189-203.

94. Tucker KL, Smith CE, Lai CQ, et al. (2013) Quantifying diet for nutrigenomic studies. Annu Rev Nutr 33, 349-371.

95. Lloyd AJ, Beckmann M, Haldar S, et al. (2013) Data-driven strategy for the discovery of potential urinary biomarkers of habitual dietary exposure. Am J Clin Nutr 97, 377-389.

96. Vernocchi P, Vannini L, Gottardi D, et al. (2012) Integration of datasets from different analytical techniques to assess the impact of nutrition on human metabolome. Front Cell Infect Microbiol 2, 156. 